\title{
Electronic Structure Modification of Boron and Nitrogen Ion-Implanted Graphene Fingerprinted by STEM-EELS
}

\author{
D.M. Kepaptsoglou ${ }^{1}$, C.R. Seabourne ${ }^{2}$, T. Hardcastle ${ }^{2}$, R. Nicholls ${ }^{3}$, W. Pierce ${ }^{4}$, R. Zan ${ }^{4}$, U. Bangert ${ }^{4,5}$, \\ A.J. Scott ${ }^{2}$ and Q.M. Ramasse ${ }^{1}$
}

${ }^{1}$ SuperSTEM Laboratory, STFC Daresbury Campus, United Kingdom

${ }^{2}$ Institute for Materials Research, SPEME, University of Leeds, Leeds, United Kingdom

${ }^{3}$ Department of Materials, University of Oxford, Oxford, United Kingdom

${ }^{4}$ School of Materials, University of Manchester, Manchester, M13 9PL, United Kingdom

${ }^{5}$ Department of Physics and Energy, University of Limerick, Limerick, Ireland

In the past few years, graphene, boron nitride and other 2D materials have stirred a research frenzy in materials science. In addition to their very promising application prospects, the field of $2 \mathrm{D}$ materials has also become a remarkably fruitful 'playground' for physicists, allowing them to use newly developed capabilities of electron microscopes [1] to study the structure-property relationships of materials literally one atom at a time. From imaging, to single dopant chemical and electronic structure determination, atom-by-atom fingerprinting of 2D materials is now possible thanks in large parts to advances in aberration correction and to 'gentle' observation conditions such as low-dose techniques [1-7].

Building on pioneering work in the application of STEM-based spectroscopy in the study of 2D materials [2,4-7] we present results on the modification of the electronic structure of graphene, by single $\mathrm{N}$ or $\mathrm{B}$ dopant atoms. The introduction of $\mathrm{N}$ or $\mathrm{B}$ atoms in the graphene lattice is predicted to have a drastic effect on graphene's band structure, such as the opening of an optical bandgap and resulting in ntype or p-type doping, respectively, with carrier concentrations allowing practical transistor applications. In our samples, the introduction of the dopants in the graphene was achieved by low energy ion implantation [7,8], a technique commonly used by the modern semiconductor industry and which has the potential to revolutionize graphene technology. High Angle Annular Dark Field imaging and atomically resolved electron energy loss spectroscopy were performed in a Nion UltraSTEM100 dedicated STEM instrument and were used to unambiguously identify single dopant atoms (Figure 1) and determine the doping levels as a function of ion implantation energy and flux.

Furthermore, the electronic structure modifications due to the single dopant B or N atoms, are strikingly demonstrated by a clear signature in the near-edge fine structure of the B and N EELS $K$ edges but also that of neighboring $\mathrm{C}-\mathrm{K}$ atoms (Figure 1). Ab initio calculations are used simulate experimental spectra (Figure 2) and to rationalize the experimental observations, thus providing further insight into the nature of bonding around the foreign species [9].

\section{References}

[1] O.L. Krivanek, M.F. Chisholm, M. Murfitt et al., Ultramicroscopy 123 (2012), p. 90.

[2] K. Suenaga, Y. Sato, Z. Liu et al., Nature Chem 1 (2009), p. 415-418.

[3] T. C. Lovejoy, Q.M. Ramasse, M. Falke et al., Appl. Phys. Lett. 100 (2012), 154101.

[4] Q. M. Ramasse, C. R. Seabourne, D.M. Kepaptsoglou et al., Nano Lett. 12 (8) (2012), p. 3936.

[5] W. Zhou, M. D. Kapetanakis, M.P. Prange et al., Phys. Rev. Lett. 109 (2012), 206803.

[6] R.J. Nicholls, A.T. Murdock, J. Tsang et al., ACS Nano 7 (2013), p. 7145.

[7] U. Bangert, W. Pierce, D.M. Kepaptsoglou et al., Nano Letters 13 (2013), p. 4902. 
[8] Y. Xu, K. Zhang, C. Brüsewitz et al., AIP Advances 3 (2013), p. 072120.

[9] SuperSTEM is the UK National Facility for Aberration-Corrected STEM and is funded by the UK Engineering and Physical Sciences Research Council (EPSRC)
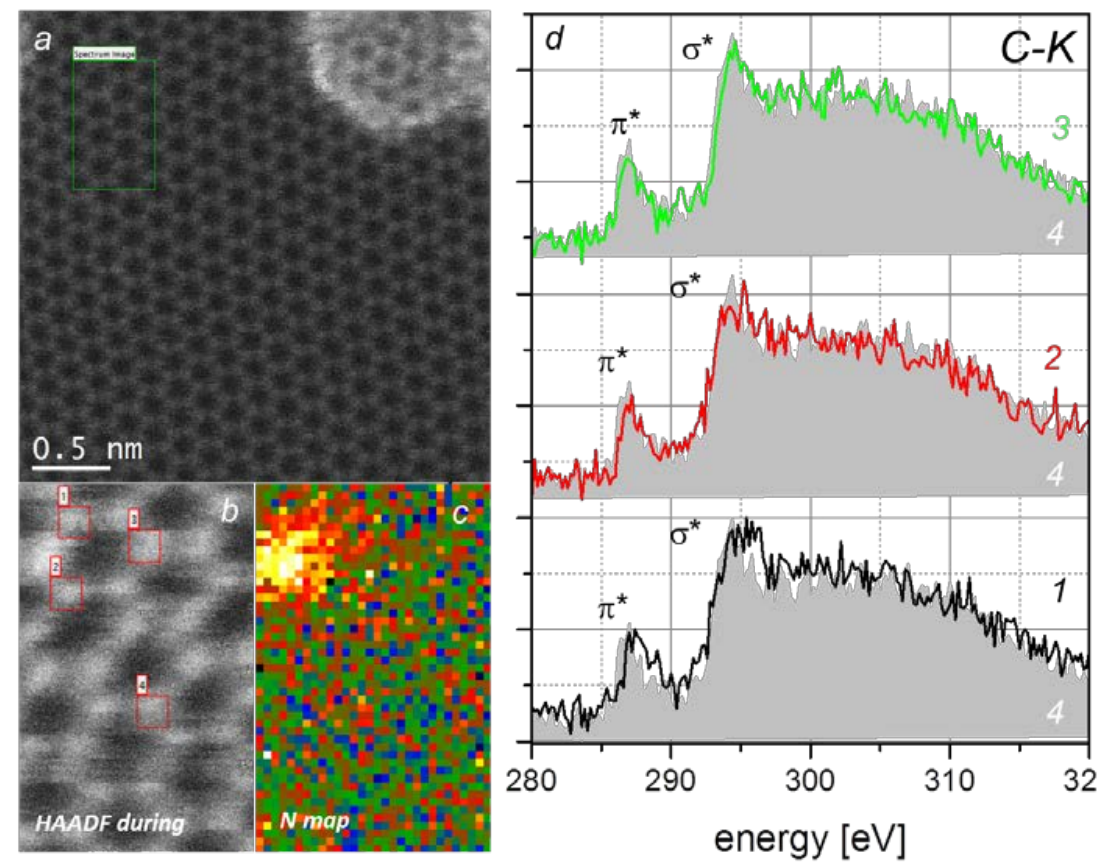

Figure 1. a) HAADF survey image of N-implanted graphene, b) HAADF signal and c) atomically resolved N-K EELS map, showing a single $\mathrm{N}$ dopant in the graphene lattice. d) C-K edge EELS spectra from neighboring $\mathrm{C}$ atoms, showing changes in the near-edge fine structure.
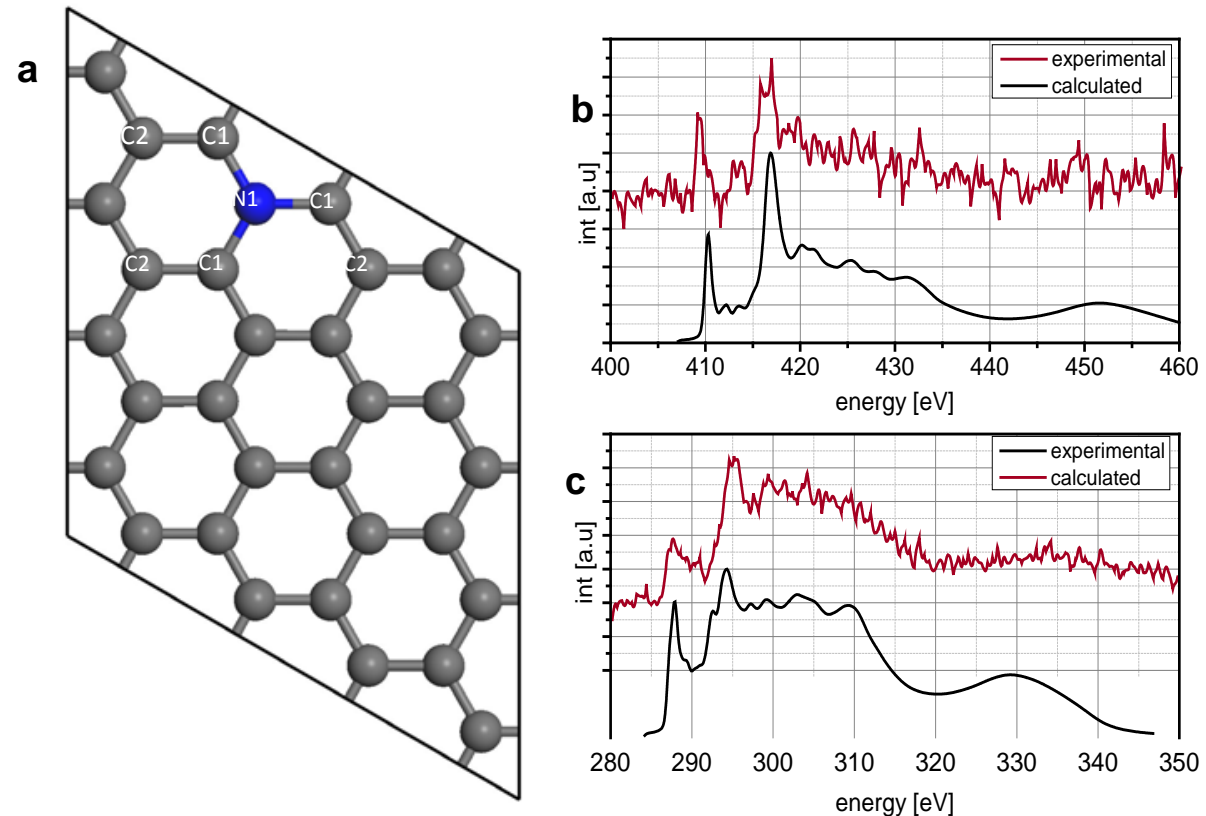

Figure 2. a) Supercell used for the simulations of EELS spectra (carried out by CASTEP), and experimental and simulated $\mathrm{N}-K$ edge of the single $\mathrm{N}$ dopant atom and c) $\mathrm{C}-K$ edge of the first (C1) $\mathrm{C}$ atom neighbor. 